Elsevier required licence: (C) <2018>. This manuscript version is made available under the CC-BY-NC-ND 4.0 license http://creativecommons.org/licenses/by-nc-nd/4.0/ 


\title{
Low-energy Structures Embedded With Smart Dampers
}

\author{
Quang Ha*, Sayed Royel, Carlos Balaguer \\ University of Technology Sydney, 15 Broadway, Ultimo NSW 2007, Australia \\ Universidad Carlos III de Madrid, Calle Madrid, 126, 28903 Getafe, Madrid, Spain
}

\begin{abstract}
Building structures, subject to dynamic loadings or external disturbances, may undergo destructive vibrations and encounter different degrees of deformation. Modeling and control techniques can be applied to effectively damp out these vibrations and maintain structural health with a low energy cost. Smart structures embedded with semi-active control devices, offer a promising solution to the problem. The smart damping concept has been proven to be an effective approach for input energy shaping and suppressing unwanted vibrations in structural control for buildings embedded with magnetorheological fluid dampers (MRDs). In this paper, the dissipation energy in MRD is studied by using results from induced hysteretic effect of structural vibrations while the fluid is placed under a controlled magnetic field. Then, a frequency-shaped second-order sliding mode controller (FS2SMC) is designed along with a low-pass filter to implement the desired dynamic sliding surface, wherein the frequency responses of the hysteretic MRD is represented by its magnitude and phase describing functions. The proposed controller can thus shape the frequency characteristics of the equivalent dynamics for the MRD-embedded structure against induced vibrations, and hence, dissipate the energy flow within the smart devices to prevent structural damage. Simulation results for a 10-floor building model equipped with current-controlled MRDs, subject to horizontal seismic excita-
\end{abstract}

\footnotetext{
* Corresponding author

Email addresses: quang.ha@uts.edu.au (Quang Ha), sayed.royel@uts.edu.au (Sayed Royel), balaguer@ing.uc3m.es (Carlos Balaguer)
}

Preprint submitted to Journal of Energy and Buildings

July 9, 2018 
tions validate the proposed technique for low-energy structures with smart devices. The closed-loop performance and comparison in terms of energy signals indicate that the proposed method allows not only to reduce induced vibrations and input energy, but also its spectrum can be adjusted to prevent natural modes of the structure under external excitations.

Keywords: Low-energy structure, smart damping, energy dissipation, modeling and control, frequency-shaped sliding mode, magnetorheological damper.

\section{Introduction}

Analysis of life cycle cost for energy-efficient buildings is evaluated based on energy consumption, assessment of environmental impact or natural hazards, and prediction of structural or non-structural damage [1]-[2]. Various elements 5 equipped with energy-efficient features of the engineering structures likely experience different levels of damage subject to external dynamic loadings such as seismic events or gusty winds, depending on the specific geographic region where the structures are situated [3]. Thereby, it may increase future costs associated with post-event repair or replacement to maintain structural health or reinstate an acceptable level. Studies have shown that cumulative damage cost can be higher than energy-efficient features and accordingly payback time for building energy efficiency investment will be prolonged [1]-[3].

Modern structures involve not only energy management [4, 5] but also condition assessment and safety management, whereby the integration of modeling, control and health monitoring is of crucial importance [6]. In quake-prone areas, building structures often undergo vibrations in response to the ground motion caused by the seismic energy and fail to dissipate inelastic energy due to excessive lateral motion, resulting in structural deformation [7, [8]. Moreover, taller, slimmer and lighter structures using high-strength materials with the same modulus of elasticity, i.e. less stiff structures, may make them more prone to dynamic loading sources, which cause discomfort and eventually, structural deterioration 
[9. Thus, adequate strength and energy dissipation capacity should be rendered in the structure to limit the overall structural motion and shift away its natural frequency from the resonance region under the disturbance excitation to maintain the structural health at a controllable embodied energy level. For example, the structural stiffness and damping can be adjusted whilst keep the amount of material utilized to a minimum.

It is possible to increase the stiffness of a building through selecting an appropriate structural configuration. Damping can be increased through the installation of auxiliary robust damping devices, since the damping characteristics, such as inherent damping, of the core structural system is relatively ambiguous until the building is completed [10. Alternatively, damping from external devices can be promising thanks to the extensive research conducted in the last decades, which make them a competent solution for mitigating the structural vibration problems in any dynamic application. However, active devices require a large supply of energy, for example in active mass dampers.

Energy-dissipative semi-active devices, such as the magnetorheological (MR) fluid damper (MRD) [11, 12], MR elastomer base isolator [13, MR pin joint [13] provide supplementary robust damping for the attenuation of vibrations induced

40 by excitation sources into the structure. The semi-active control systems can dissipate vibration energy into heat through the adjustment of damping and stiffness characteristics of the system under a low-power control signal and failsafe operations. The controlled damping forces always oppose the motion of the structure, hence, promoting stability, as well as reducing the consequence 45 of system uncertainties. 9 , 10.

The level of possible damage of individual structural members, e.g., beams, columns, and roof/floor slabs can be determined by the transmitted external dynamic loading into structural vibrations. The induced energy can then be decomposed into different forms, i.e. kinetic, damping, recoverable elastic strain and irrecoverable hysteretic dissipation in the structure during a loading event [14, [15], 7]. Semi-active control with MR fluid devices provides energy-efficient protection of engineering structures [1]-[18 by dissipating excess energy into 
heat through the fluid. This heat is then transferred to the environment by convection and/or conduction [17]-20].

For analysis of the energy flow, excitations can be represented in the frequency domain by their power spectral density functions and dynamic behavior of the structures are characterized by their frequency response functions (FRFs). The frequency domain approach to structural control allows for a roll-off of the control action at high frequencies and specify the disturbance attenuation over desired bands. The frequency-shaping (FS) technique to the linear-quadratic (LQ) design was first proposed in [21] with the cost functional expressed via the frequency variable $\omega$. A discrete time approach to the frequency-shaping LQ control using the Parseval's theorem was reported in 22] for active suspension system. A challenging requirement for these structural control systems remains strong robustness in face of system uncertainties and large disturbances. For this, sliding mode control (SMC) is known as a discontinuous robust control [23][25], which forcibly confines the system's states to a user-chosen sliding surface by varying the control structure in the state space. To extend the SMC design to the frequency domain, frequency-shaped SMC (FSSMC) has been developed and applied to various mechanical systems including flexible robot manipulators [26], 27], active vibration control [28], and hard disk drives [29].

In FSSMC, the sliding surface is obtained by applying a desired linear operator to the original sliding function for shaping the system equivalence dynamics in the frequency domain [30, 28]. An output feedback FSSMC was studied in

75 31] for damping out structural vibrations of a smart flexible cantilever beam, where the system states are implicitly obtained by measuring the output at a faster rate than the control input. The works mentioned above have not clearly explained on the dissipation of vibration-induced energy in the controlled smart devices. To date the analysis of the energy flow in the structures has not been directly addressed for control and monitoring to achieve energy-efficient embedded structures under vibrations induced from external loadings. Here, the frequency domain advantage is taken into account within a modelling and control framework to analyze the energy relationships of the smart devices in the 
structures, and to design a robust controller to achieve low-energy and resilient structures against dynamic loadings such as earthquakes or gusty winds.

This paper is organized as follows. After the introduction, the properties of an MRD and energy equations of smart devices in building structure are studied in Section 2. Using the experimental test data, describing functions (DFs) of magnitude and phase responses are obtained to interpret the intrinsic currentdependent hysteretic dynamics of the MRD. A frequency-shaped second-order SMC (FS2SMC) is proposed and designed for buildings embedded with smart devices in in Section 3 . Section 4 presents the application and simulation results obtained with the proposed approach for a 10-floor building. Section 5 presents a discussion on the energy flow in the structure embedded with smart devices 95 in the presence of external disturbances. The energy spectra of the structure are compared between the uncontrolled case, the Lyapynov-based control and the proposed FS2SMC in terms of kinetic, damping, strain, and input energy signals to illustrate the capability of a low-energy smart structure in suppressing quake-induced vibrations. A conclusion is finally drawn in Section 6 .

\section{Low-energy Structure embedded with Smart Devices}

The smart device considered in this section is the magnetorheological damper (MRD). To experimentally study the dissipation and energy-related aspects in the device for analysis of low-energy resilient structures embedded with MRDs, the RD-8041-1 damper manufactured by LORD Corporation is characterized by using a thermal camera.

\subsection{Energy cycle of an $M R D$}

The damping capacity of smart structures embedded with MRDs depends on the amount of energy dissipated in the devices as a result of their induced hysteretic effect, during a typical vibration cycle while the MRDs operate at a constant magnetic field [17, [18]. Let $c_{\mathrm{MR}}, E_{\mathrm{MR}}, f_{\mathrm{MR}}, k_{\mathrm{MR}}$, and $\zeta_{\mathrm{MR}}$ denote the equivalent damping, dissipated energy, output force function, effective stiffness 


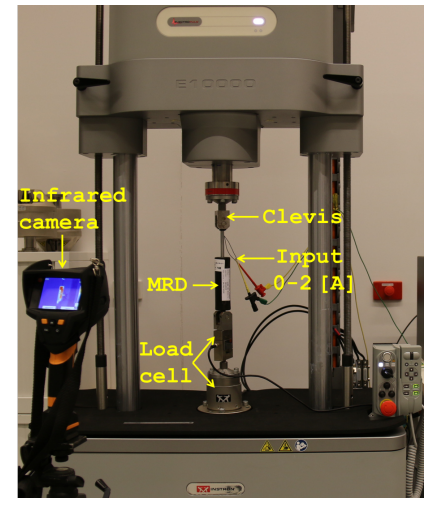

(a)

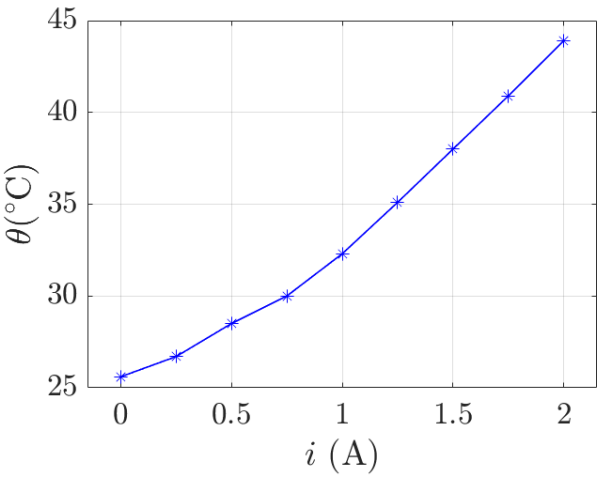

(b)

Figure 1: RD-8041-1 test system and identified temperature versus magnetization current.

and damping ratio of the device. At frequency $f$, the MRD dissipates its cyclic energy in the magnetorheological fluid via the formation of a closed-loop mechanical lag in the force-displacement trajectory. This energy can be expressed as

$$
E_{\mathrm{MR}}=\oint f_{\mathrm{MR}} d x=2 \pi^{2} f c_{\mathrm{MR}} E^{2}=2 \pi k_{\mathrm{MR}} \zeta_{\mathrm{MR}} E^{2},
$$

where $f_{\mathrm{MR}}=c_{\mathrm{MR}} \dot{x}$, the equivalent damping coefficient $c_{\mathrm{MR}}=k_{\mathrm{MR}} \zeta_{\mathrm{MR}} /(\pi f)$, $x=E \sin (\omega t)$ is the sinusoidal displacement of amplitude $E$ and angular frequency $\omega=2 \pi f$. The derivation of (1) is given in Appendix. To characterize this hysteretic force-velocity relationship, the MRD is mounted on a Schenck machine, the Instron ElectroPuls ${ }^{\mathrm{TM}}$ E10000, as shown in Fig. 1(a).

To record the dissipation of the damper RD-8041-1 in response to the magnetic field strength at a magnetization current $i(t)$, we used a Testo $875-2 \mathrm{i}$ thermal camera. The mechanical energy dissipated in the magnetorheological fluid inside the damper housing is converted into heat, depending on the magnetic field strength. Thus, an increase in the magnetization current $i$ will result in a temperature rise in the fluid inside the MRD housing, as depicted in Fig. 1(b). Taking into account also the Joule effect of the coil resistance $R$, the power $P$ 
of the system can be given by

$$
P=-f_{\mathrm{MR}}(t) \dot{x}-i^{2}(t) R
$$

where $R=5 \Omega(7 \Omega)$ at the ambient temperature (at $71^{\circ} \mathrm{C}$ ) and $i \in[02]$ A for the MRD RD-8041-1 used in experiments.

Figure 2 shows that hysteresis occurring in the damper's dynamic relation between input-output (I/O) variables represents memory effects involving fielddependent friction in the MR fluid suspension, leading to energy dissipation. Notably, in both the force-displacement (Fig. 2(a)) and force-velocity (Fig. 2(b)) relationships, hysteresis loops representing intrinsic nonlinearity and complex dynamics of the damper are not zero-centered, which account for the effect of the accumulator at the bottom of the MRD.

The time responses of the energy and power (energy rate) obtained for the hysteresis loops of the conjugate force-displacement and force-velocity trajectories of Figs. 2(a) and (b) are shown in Figs. 3(a) and (b), respectively. They indicate that the force-displacement hysteresis progresses along clockwise tra125 jectories while the force-velocity hysteresis follows anticlockwise paths. It can be interpreted from these figures that the MRD attached to a structure stores its elastic energy of the structure via its spring component and when the structural motion and correspondingly the MRD stroke is to reverse their direction, the damper would transfer the energy back to the structure. The energy alterations can be seen at the enclosed area of quadrants II and IV in Figs. 2(b) and 3(b), that is $-f_{\mathrm{MR}} \cdot \dot{x}$. The corresponding negative values occur in only a short duration, $\Delta t_{\mathrm{II}}+\Delta t_{\mathrm{IV}}=0.042+0.048=0.09 \mathrm{~s}$, about $18 \%$ of one vibration period $\frac{2 \pi}{\omega}=0.5 \mathrm{~s}$, as shown in Fig. 3(b). The cyclic dissipation and energy rate of the smart device not only depend on the oscillation amplitude of the MRD stroke, but also vary with respect to the applied magnetization current and excitation frequency, as shown in Figs. 3(c) and (d), respectively. In the following, describing function models are developed for the MRD to comprehensively capture these complicated relations 13 . 


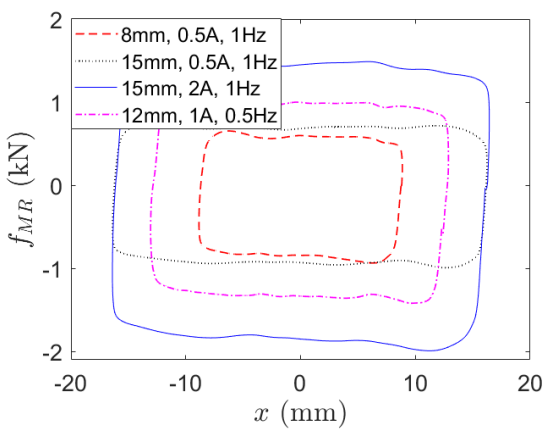

(a) $f_{\mathrm{MR}}$ vs $x$ trajectories.

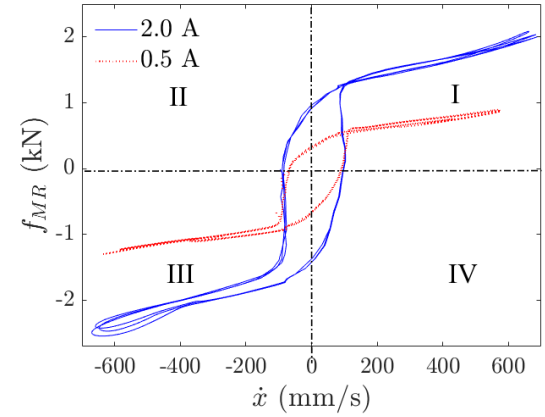

(b) $f_{\mathrm{MR}}$ vs $\dot{x}$ trajectories.

Figure 2: Measured RD-8041-1 hysteresis.

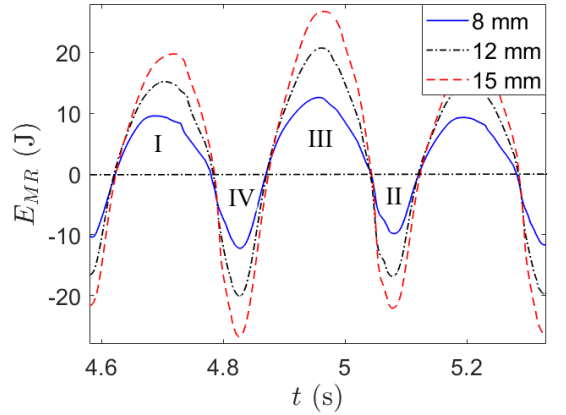

(a) One energy cycle, $2 \pi / \omega=0.5 \mathrm{~s}$.

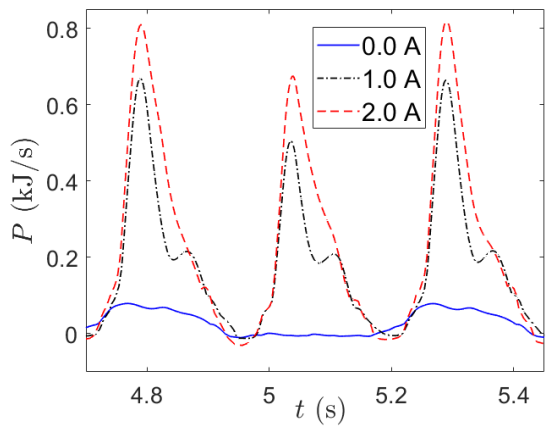

(c) $x=8 \sin (2 \times 2 \pi t) \mathrm{mm}$.

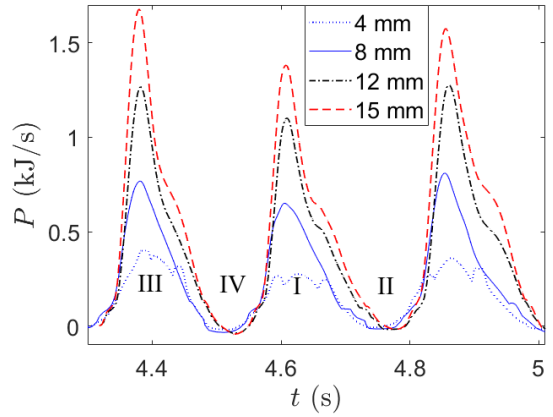

(b) $x=E \sin (2 \times 2 \pi t) \mathrm{mm}, i=2 \mathrm{~A}$.

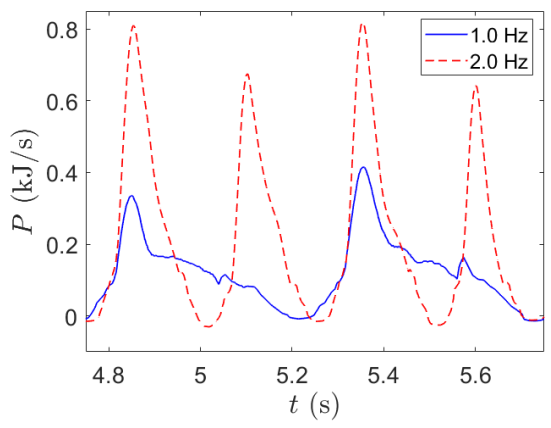

(d) $x=8 \sin (2 \pi f t) \mathrm{mm}, i=2 \mathrm{~A}$.

Figure 3: MRD (a) dissipated energy and (b)-(d) energy rate during a vibration cycle operated at a constant magnetic field: $i \in[02] \mathrm{A}, E=[4,8,12,15] \mathrm{mm}, f=[0.5,1,2,3] \mathrm{Hz}, R=5 \Omega$, $\theta \in[25.643 .9]^{\circ} \mathrm{C}$. 


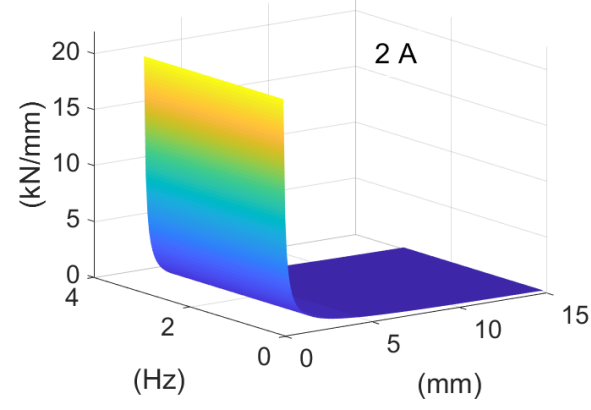

(a) DF gain at $i=2 \mathrm{~A}$.

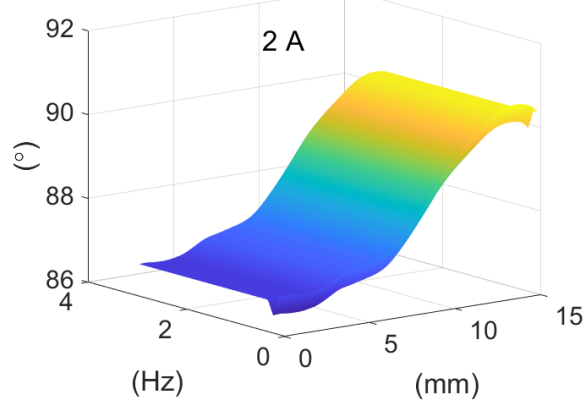

(b) DF phase at $i=2 \mathrm{~A}$.

Figure 4: RD-8041-1 hysteresis DF magnitude and phase.

\subsection{MRD describing function model} zation data. In general, the gain $N(E, f, i)$ decreases as the amplitude $E$ and/or frequency $f$ increases. On the other hand, the DF gain of MRD increases with the magnetization $i$ within its operational range.

Since the DF is a complex quantity, i.e. $N e^{j \phi}, \mathrm{DF}$ of MRD exhibits a phase shift $\phi$ as shown in Fig. 4(b). A rational approximation technique yields the following expressions [13]:

$$
\begin{aligned}
& N(E, f, i)=\frac{n_{0}+n_{1} i+n_{2} i^{2}}{E+m_{0}+m_{1} f}=\frac{0.102+2.23 i-1.08 i^{2}}{E-0.33+0.24 f}, \\
& \phi(E, f, i)=\frac{h_{0}+h_{1} i+h_{2} i^{2}}{E+g_{0}+g_{1} f}=\frac{-0.7+7.6 i-2.9 i^{2}}{E+1.9-0.08 f},
\end{aligned}
$$

where current $i \in\left[\begin{array}{ll}0 & 2\end{array}\right] \mathrm{A}$, frequency $f \in\left[\begin{array}{lll}0.1 & 3\end{array}\right] \mathrm{Hz}$, and displacement amplitude 150

$E \in[0.120] \mathrm{mm}$. 


\subsection{Energy balance equation of buildings with smart devices}

The problem of energy balance in a building structure embedded with smart dampers is considered in this section. Since internal forces within an engineering structure can be derived using relative displacements and velocities, we herein compute the input energy in terms of the relative motion.

Given an $n$ degree-of-freedom (dof) shear structure of mass $M$, stiffness $K$, and viscous damping $C$, embedded with $n$ MRDs subject to dynamic loading sources with acceleration vector $\ddot{x}_{g}$, the governing equation can be described by

$$
M \ddot{x}+C \dot{x}+K x=\Gamma f_{\mathrm{MR}}(x, i)-M \ddot{x}_{g},
$$

where $\Gamma \in \mathbb{R}^{n \times n}$ is a factor matrix taking into account the location and number of MRDs; $f_{\mathrm{MR}}(x, i)$ is the controllable damping force vector; $x=r-x_{g}$ denotes the relative displacement between the ground and each mass as shown in Fig. 5(a). Here, $r$ and $x_{g}$ are vectors of the absolute displacements of the floors and ground with respect to a reference frame $x x-y y$ and an inertial frame $x x-y y^{\prime}$, respectively. As shown in Fig. 5(b), the intelligent devices are rigidly mounted to the fixtures between each floor, and the midpoint of each damper coincides with the intersection of axes $x x$ and $y y$, i.e. $x_{k}=x_{d k}$ for the $k$-th floor.

Suppose the model (4) satisfies the following assumptions:

(A1.) $M^{-1}$ exists and $M^{-1} K$ has a set of $n$ linearly independent eigenvectors $v_{1}, \ldots, v_{n}$

(A2.) the unknown dynamic disturbance $f_{d}(t)=-M \ddot{x}_{g}$ and its timederivative are bounded for $t \in[0 \infty)$,

(A3.) zero initial conditions, i.e., $x\left(t_{0}\right)=0, \dot{r}\left(t_{0}\right)=0$, and

(A4.) $x=\left[x_{1}, \ldots, x_{n}\right]^{\mathrm{T}}, \dot{x} \in \mathbb{R}^{n}$ and $\ddot{x} \in \mathbb{R}^{n}$ are available for on-line measurement.

By integrating both sides of (4) over the structural response path from $t_{0}$ 


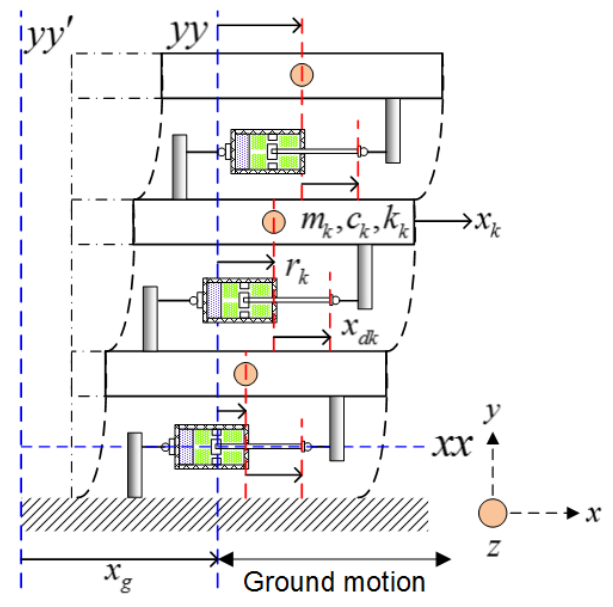

(a)

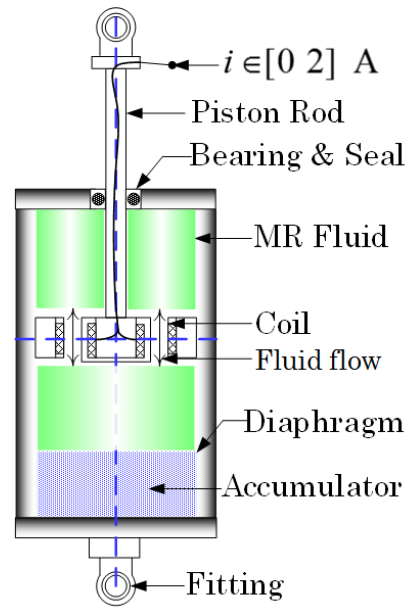

(b)

Figure 5: Smart building integrated with energy-dissipative devices; $x_{d k}=x_{k}, k \in[1, n]$.

(when the ground motion excitation starts) to $t$, we obtain:

$$
\begin{aligned}
& \int_{x\left(t_{0}\right)}^{x(t)} \ddot{x}^{\mathrm{T}} M d x+\int_{x\left(t_{0}\right)}^{x(t)} \dot{x}^{\mathrm{T}} C d x+\int_{x\left(t_{0}\right)}^{x(t)} x^{\mathrm{T}} K d x=\int_{x\left(t_{0}\right)}^{x(t)} f_{\mathrm{MR}}^{\mathrm{T}} \Gamma^{\mathrm{T}} d x-\int_{x\left(t_{0}\right)}^{x(t)} \ddot{x}_{g}^{\mathrm{T}} M d x \\
& \int_{x\left(t_{0}\right)}^{x(t)} \dot{x}^{\mathrm{T}} M d \dot{x}+\int_{t_{0}}^{t} \dot{x}^{\mathrm{T}} C \dot{x} d t+\int_{x\left(t_{0}\right)}^{x(t)} x^{\mathrm{T}} K d x=\int_{t_{0}}^{t} f_{\mathrm{MR}}^{\mathrm{T}} \Gamma^{\mathrm{T}} \dot{x} d t-\int_{t_{0}}^{t} \ddot{x}_{g}^{\mathrm{T}} M \dot{x} d t \\
& \frac{1}{2} \dot{x}^{\mathrm{T}} M \dot{x}+\int_{t_{0}}^{t} \dot{x}^{\mathrm{T}} C \dot{x} d t+\frac{1}{2} x^{\mathrm{T}} K x=\int_{t_{0}}^{t} f_{\mathrm{MR}}^{\mathrm{T}} \Gamma^{\mathrm{T}} \dot{x} d t-\int_{t_{0}}^{t} \ddot{x}_{g}^{\mathrm{T}} M \dot{x} d t \\
& E_{k}+E_{\zeta}+E_{s}=E_{\mathrm{MR}}-E_{i},
\end{aligned}
$$

where $E_{k}, E_{\zeta}, E_{s}$, and $E_{i}$ represent the relative kinetic, damping, strain, and input energies, respectively. The absolute kinetic and input energies can also be defined respectively by $\frac{1}{2} \dot{r}^{\mathrm{T}} M \dot{r}$ and $\int_{t_{0}}^{t} \ddot{r}^{\mathrm{T}} M \dot{x}_{g} d t$. Consequently, the residual absolute energy terms can be derived in the same way.

\section{Frequency-shaped Structural Control}

After having established the energy relations a building structure embedded with smart dampers, we now proceed with the robust control design to inject a a small amount of control energy to dissipate vibration energy induced by external 
In order to dynamically shape the frequency response of the equivalent dynamics, the sliding function is cast by using a dynamic linear operator $L(s)$, a function of $s$, instead of $\rho_{k}$. We design the dynamic sliding surface with a second-order low-pass filter (LPF) as

$$
\sigma=\mathcal{L}(s) z=\sum_{k=1}^{n-1} L(s) z_{k}+z_{n}, \mathcal{L}(s)=[L(s), \ldots, L(s), 1]
$$

for example, to get a steeper roll-off of $|L(j \omega)|, \omega \in[0, \infty)$ for large values of frequency $\omega$. Herein, $L(s)=\frac{b_{0}}{s^{2}+a_{1} s+a_{0}}$ gives a $|L(j \omega)|$ with an asymptotic slope of $-40 \mathrm{~dB} /$ decade above cut-off frequency. The algebraic manipulation of (6) 
gives

$$
\begin{aligned}
\sigma & =\frac{b_{0}}{s^{2}+a_{1} s+a_{0}} z_{1}+\cdots+\frac{b_{0}}{s^{2}+a_{1} s+a_{0}} z_{n-1}+z_{n} \\
\Rightarrow & \ddot{\sigma}+\sigma\left(a_{1} s+a_{0}\right)=b_{0} \sum_{k=1}^{n-1} z_{k}+\ddot{z}_{n}+z_{n}\left(a_{1} s+a_{0}\right) \\
\Rightarrow & \ddot{\sigma}=b_{0} \sum_{k=1}^{n-1} z_{k}+\ddot{z}_{n}-\left(a_{1} s+a_{0}\right)\left(\frac{b_{0} \sum_{k=1}^{n-1} z_{k}}{s^{2}+a_{1} s+a_{0}}\right) \\
\Rightarrow & \ddot{\sigma}=b_{0}\left(\frac{s^{2}}{s^{2}+a_{1} s+a_{0}}\right) \sum_{k=1}^{n-1} z_{k}+\frac{\partial h}{\partial z} \dot{z}+\frac{\partial g}{\partial z} \dot{z} u+g(z) \dot{u} .
\end{aligned}
$$

Now, we have

$$
\ddot{\sigma}=s^{2} L(s) z_{1}+\cdots+s^{2} L(s) z_{n-1}+H(z, u)+g(z) v,
$$

where $H=\frac{\partial h}{\partial x} \dot{z}+\frac{\partial g}{\partial x} \dot{z} u$ and $v=\dot{u}$ is denoted as the new control variable 33. Then, we can derive the best approximation of the continuous control law that achieves $\ddot{\sigma}=0$ :

$$
\hat{v}=-\frac{s^{2} L(s) z_{1}+\cdots+s^{2} L(s) z_{n-1}+\hat{H}}{\hat{g}},
$$

where $\hat{g}$ and $\hat{H}$ are the nominal models of $g$ and $H$, respectively. A reaching control input $v_{R}$ is added to $\hat{v}$ to ensure that the plant dynamics reach the sliding surface in finite time [25. Substituting $v=\hat{v}+v_{R}$ into (7) results in [34:

$$
\begin{aligned}
\ddot{\sigma} & =\sum_{k=1}^{n-1} s^{2} L(s) z_{k}+H-\frac{g(z)}{\hat{g}(z)} \sum_{k=1}^{n-1} s^{2} L(s) z_{k}-\frac{g(z)}{\hat{g}(z)} \hat{H}+g(z) v_{R} \\
& =H-\frac{g(z)}{\hat{g}(z)} \hat{H}+\sum_{k=1}^{n-1} s^{2} L(s) z_{k}\left[1-\frac{g(z)}{\hat{g}(z)}\right]+g(z) v_{R}=\delta(z)+g(z) v_{R},
\end{aligned}
$$

where $\delta(z):=H-\frac{g(z)}{\hat{g}(z)} \hat{H}+\sum_{k=1}^{n-1} s^{2} L(s) z_{k}\left[1-\frac{g(z)}{\hat{g}(z)}\right]$.

Suppose the perturbation term $\delta(z)$ satisfies the inequality

$$
\left|\frac{\delta(z)}{g(z)}\right| \leq \varrho(z)
$$

for some known positive definite function $\varrho(z)$. With $V=\frac{1}{2} \dot{\sigma}^{2}$ chosen as a Lyapunov function candidate for (7), the time derivative of $V$ can be computed 
as

$$
\dot{V}=\dot{\sigma} \ddot{\sigma}=\dot{\sigma}\left[\delta(z)+g(z) v_{R}\right] \leq g(z)|\dot{\sigma}| \varrho(z)+g(z) \dot{\sigma} v_{R}
$$

To achieve the control objective, the robust signal $v_{R}$ is selected as

$$
v_{R}=-\beta(z) \operatorname{sgn}(\dot{\sigma})-\kappa \dot{\sigma}, \beta(z) \geq \varrho(z)+\eta,
$$

where $\kappa>0, \eta>0$, so that the term $g(z) \dot{\sigma} v_{R}$ is negative and dominates over the residual term $g(z)|\dot{\sigma}| \varrho(z)$ when $\dot{\sigma} \neq 0$, giving the net results to force $|\dot{\sigma}|$ to reach zero. Finally, we have

$$
v=-\frac{s^{2} L(s) z_{1}+\cdots+s^{2} L(s) z_{n-1}+\hat{H}}{\hat{g}(z)}-\beta(z) \operatorname{sgn}(\dot{\sigma})-\kappa \dot{\sigma} .
$$

Substituting (11) into 10 yields

$$
\begin{aligned}
\dot{V} & \leq g(z)|\dot{\sigma}| \varrho(z)-g(z) \beta(z) \dot{\sigma} \operatorname{sgn}(\dot{\sigma})-g(z) \kappa \dot{\sigma}^{2} \\
& \leq g(z)|\dot{\sigma}| \varrho(z)-g(z)(\varrho(z)+\eta)|\dot{\sigma}|-g(z) \kappa \dot{\sigma}^{2}=-g(z) \eta|\dot{\sigma}|-g(z) \kappa \dot{\sigma}^{2} \\
& \leq-g_{0} \eta|\dot{\sigma}|-g_{0} \kappa \dot{\sigma}^{2} \leq-g_{0} \kappa \dot{\sigma}^{2}=-2 g_{0} \kappa V .
\end{aligned}
$$

200

By integrating the differential inequality over the time interval $t_{0} \leq \tau \leq t$, we obtain $V(t) \leq V\left(t_{0}\right) e^{-2 \kappa g_{0}\left(t-t_{0}\right)}$. Thus, $V(t)$ will tend to zero exponentially where $\kappa$ is the decay rate at which the sliding surface is attained. From 10 and 13], we obtain $\dot{\sigma} \ddot{\sigma} \leq-g_{0} \eta|\dot{\sigma}|-g_{0} \kappa \dot{\sigma}^{2}$. Since $g_{0} \kappa \dot{\sigma}^{2} \geq 0$, and by neglecting the nonlinear term, we also have $\frac{d}{d t}|\dot{\sigma}(t)| \leq-g_{0} \kappa|\dot{\sigma}(t)| \Rightarrow|\dot{\sigma}(t)| \leq\left|\dot{\sigma}\left(t_{0}\right)\right| e^{-\kappa g_{0}\left(t-t_{0}\right)}$ to substantially reduce the amplitude of the switching term in the control and hence, the commonly encountered chattering problem associated with sliding mode control.

\subsection{Smart structural control}

To further implement the proposed control strategy, described in the previous section, to low-energy MRD-embedded structures, a modal transformation is first applied to the structure dynamics, e.g. of a multi-floor building. Thus, with the transform $x=\Phi q$ ( $\Phi$ is a nonsingular transformation matrix) for the 
modal coordinate vector $q$ [28, we can obtain a set of $n$ second-order motion equations decoupled from (4) for each mode, $m \in[1, n]$, as:

$$
\ddot{q}_{m}+2 \zeta_{m} \omega_{m} \dot{q}_{m}+\sum_{r=1, r \neq m}^{n} \mu_{m r} \dot{q}_{r}+\omega_{m}^{2} q_{m}=u_{m}+d_{m},
$$

where $\omega_{m}, \zeta_{m}, u_{m}, q_{m}, d_{m}$, and $\mu_{m r}$ are respectively the $m$-th modal frequency, damping ratio, entry of the modal control $u=\Phi^{-1} M^{-1} \Gamma f_{\mathrm{MR}}=\Omega f_{\mathrm{MR}}$, modal coordinate, disturbance component and the $m r$ th modal coupling term of the damping matrix. From (6) and (7), the following frequency-shaped sliding function is designed:

$$
\begin{aligned}
\sigma_{m} & =\frac{b_{0}}{s^{2}+a_{1} s+a_{0}} q_{m}+\dot{q}_{m}=L_{m}(s) q_{m}+\dot{q}_{m} \\
\Rightarrow \ddot{\sigma}_{m} & =s^{2} L_{m}(s) q_{m}-2 \zeta_{m} \omega_{m} \ddot{q}_{m}-\sum_{r=1, r \neq m}^{n} \mu_{m r} \ddot{q}_{r}-\omega_{m}^{2} \dot{q}_{m}+v_{m}+\dot{d}_{m},
\end{aligned}
$$

where $v_{m}=\dot{u}_{m}$ is the new control instead of the modal control $u_{m}$. We derive the equivalent control $\hat{v}_{m}$ to achieve $\ddot{\sigma}_{m}=0$ as follows

$$
\hat{v}_{m}=\hat{\omega}_{m}^{2} \dot{q}_{m}+2 \hat{\zeta}_{m} \hat{\omega}_{m} \ddot{q}_{m}+\sum_{r=1, r \neq m}^{n} \hat{\mu}_{m r} \ddot{q}_{r}-\hat{\dot{d}}_{m}-s^{2} L_{m}(s) q_{m},
$$

where $\hat{\omega}_{m}, \hat{\zeta}_{m}, \hat{\dot{d}}_{m}$, and $\hat{\mu}_{m r}$ are desired values chosen for the $m$-th modal frequency, modal damping, first derivative of the disturbance, and modal coupling from the damping matrix, respectively. By applying the control law (12) for $v_{m}=\hat{v}_{m}+v_{R m}$, we obtain the following FS2SMC

$v_{m}=\hat{\omega}_{m}^{2} \dot{q}_{m}+2 \hat{\zeta}_{m} \hat{\omega}_{m} \ddot{q}_{m}+\sum_{r=1}^{n} \hat{\mu}_{m r} \ddot{q}_{r}-s^{2} L_{m}(s) q_{m}-\hat{\dot{d}}_{m}-\beta_{m} \operatorname{sgn}\left(\dot{\sigma}_{m}\right)-\kappa_{m} \dot{\sigma}_{m}$,

that can ensure the condition $\dot{\sigma}_{m} \ddot{\sigma}_{m} \leq-\eta_{m}\left|\dot{\sigma}_{m}\right|-\kappa_{m} \dot{\sigma}_{m}^{2}$ as in 13, by taking

\section{Application and Simulation}

For application, we now consider a 10-storey shear building model 35] with identical values for floor mass $m_{k}=360$ tonnes, damping $c_{k}=6.2 \mathrm{MNs} / \mathrm{m}$, and 
stiffness $k_{k}=650 \mathrm{MN} / \mathrm{m}$ of each floor, $k=1,2, \ldots, 10$, that satisfies assumptions

215

\subsection{Modal decomposition and control design}

Since the mass matrix is nonsingular, (4) is written for this case as

$$
\ddot{x}+M^{-1} C \dot{x}+M^{-1} K x=M^{-1} \Gamma f_{\mathrm{MR}}+M^{-1} f_{d}, x \in \mathbb{R}^{10},
$$

whereby the diagonal and modal coupling terms of the damping matrix, i.e. $2 \zeta_{k} \omega_{k}$ and $\mu_{k r}$, can be respectively obtained, for $r \neq k$ with no dampers attached, as $\omega_{k} / 2 \pi=1.01,3.01,4.94,6.76,8.43,9.91,11.18,12.19,12.93$, $13.37 \mathrm{~Hz} ; \zeta_{k}=0.19,0.57,0.93,1.27,1.59,1.87,2.1,2.29,2.43,2.52$; and $\mu_{k r} \in\left[-1.27 \times 10^{-14}, 2.39 \times 10^{-14}\right]$. Hence, the off-diagonal damping is taken as the lower bound of the diagonal elements, i.e., $\min \left(2 \zeta_{k} \omega_{k}\right)=0.38$ while designing the FS2SMC.

Under a harmonic excitation at an angular frequency $\omega$, the controlled smart devices $\Gamma f_{\mathrm{MR}}(q, i)$ can be modelled in the frequency domain [13] as $D F_{\mathrm{MR}} Q(j \omega) \in \mathbb{C}^{10}$. Thus,

$$
\begin{aligned}
& {\left[-\omega^{2}+2 j \omega \zeta_{k} \omega_{k}+\omega_{k}^{2}\right] q_{k}(j \omega)+\sum_{r=1, r \neq k}^{10} \mu_{k r} j \omega q_{r}(j \omega)=-} \\
& {\left[\frac{\gamma_{k} N_{k} e^{j \phi_{k}}}{m_{k}} q_{k}(j \omega)+\sum_{r=1, r \neq k}^{10} \frac{\varepsilon_{k r}}{m_{k}} q_{r}(j \omega)\right]+\Upsilon F_{d}(j \omega), \Upsilon=\Phi^{-1} M^{-1},}
\end{aligned}
$$

where $\varepsilon_{k r} \in\left[0 \gamma_{k} N_{k} e^{j \phi_{k}}\right]$ denotes inter-floor damping from the MRDs mounted between the $k$-th and $(k-1)$-th floors and $\gamma_{k}$ is a factor taking into account the placement and number of MRDs. Equation 19 can be rewritten as

$$
\begin{aligned}
& {\left[-\omega^{2}+2 j \omega \zeta_{k} \omega_{k}+\omega_{k}^{2}+\frac{\gamma_{k} N_{k} e^{j \phi_{k}}}{m_{k}}\right] \Phi^{-1} x_{k}(j \omega)} \\
& +\left[\sum_{r=1, r \neq k}^{10} \mu_{k r} j \omega+\sum_{r=1, r \neq k}^{10} \frac{\varepsilon_{k r}}{m_{k}}\right] \Phi^{-1} x_{r}(j \omega)=\Upsilon F_{d}(j \omega) .
\end{aligned}
$$

Since the damping capability always takes its strongest effect at the level where the MRDs are installed, $D F_{\mathrm{MR}}$ can be considered as diagonally dominant, thus 
including only terms $\gamma_{k} N_{k} e^{j \phi_{k}}$. Other the coupling terms from the residual modes and modal decomposition errors can be lumped to disturbance $d_{k}$. Taking the assumption of Rayleigh damping [7, [15, the frequency response function (FRF) matrix, $H(j \omega)$, of the smart structure is therefore obtained as $H(j \omega)=$ $\Phi \operatorname{diag}\left(\delta_{k k}\right) \Upsilon$, where $\delta_{k k}=\frac{1}{\omega_{k}^{2}-\omega^{2}+2 j \omega \zeta_{k} \omega_{k}+\frac{\gamma_{k} N_{k} e^{j \phi_{k}}}{m_{k}}}$. Here, the transfer function of the DF model (3) is approximated as $N_{k} e^{j \phi_{k}} \simeq N_{k}\left(1+\tau_{k} s\right)$, where $\tau_{k}=$ $\frac{\phi_{0 k}}{\omega_{k} N_{0 k}}$ is the MRD equivalent time constant estimated at normalized values of amplitude $E=1$, current $i=1$ and first modal frequency $\omega_{1}=1$. Hence, the FRF of the smart structure is

$$
H(j \omega)=\sum_{k=1}^{10} \frac{\Phi_{k} \Upsilon_{k}^{\mathrm{T}}}{\omega_{k}^{2}-\omega^{2}+\frac{\gamma_{k} N_{k}}{m_{k}}+j \omega\left(2 \zeta_{k} \omega_{k}+\frac{\gamma_{k} N_{k} \tau_{k}}{m_{k}}\right)} .
$$

In our design, the controller parameters are chosen as $\kappa_{k}=10, \eta_{k}=1.25$ and $L(s)$ is a Butterworth filter, i.e. $L(s)=\frac{1}{s^{2}+1.4142 s+1}$. Notably, for the control law (17), a boundary layer [23] may be used in lieu of the signum function to smooth the response if necessary. From the modal control $u_{k}=\int v_{k}(t) d t$, the damping force can be computed as $f_{\mathrm{MR}(k)}=\Omega^{-1} u_{k}$ in which $\Omega^{-1}$ is the inverse mode participation matrix. The controllable force range should be constrained by the maximum capacity, $i_{\mathrm{M}}$ and the residual force at zero current in the passive control case. For example, the relation between the magnetization current and damping force has the following form:

$$
i_{k}=\left\{\begin{array}{cl}
\left|a_{0}+a_{1} f_{\mathrm{MR}(k)}+a_{2} f_{\mathrm{MR}(k)}^{2}\right|, & \left|i_{k}\right|<i_{\mathrm{M}}, \\
0, & \left|i_{k}\right| \geq i_{\mathrm{M}},
\end{array}\right.
$$

where $a_{0}=0.127, a_{1}=-0.00094$ and $a_{2}=0.0000021$ for the RD-8041-1 at ${ }_{225} i_{\mathrm{M}}=2 \mathrm{~A}$.

\subsection{Simulation Results}

In our simulation, four benchmark earthquake records (El-Centro 1940, Hachinohe 1968, Northridge 1994, Kobe 1995) are considered to excite the system as external disturbances. We normalized the structural dynamics and all quake records to a maximum acceleration level of $0.3 g$ so that we have 


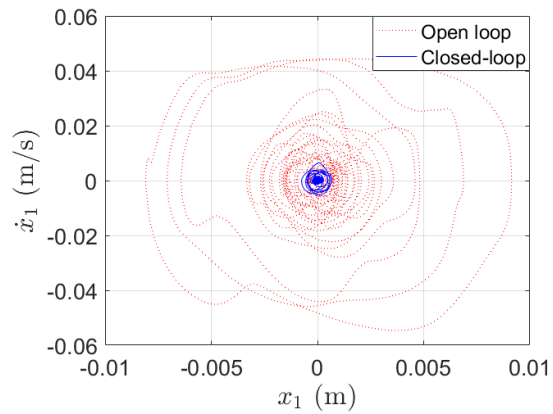

(a)

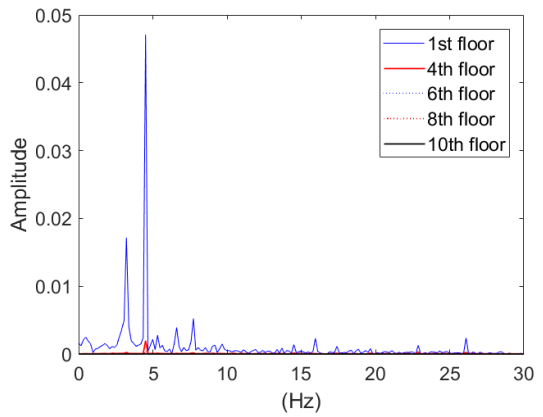

(b)

Figure 6: Closed-loop and open-loop phase portrait and the spectrum.

$x_{d k}=x_{k}<15 \mathrm{~mm}$ corresponding to the operational stroke and capacity of MRDs. Extensive simulation was conducted with the scaled-down four benchmark quake records. Typical results are shown in Fig. 6(a) for the first floor's phase portrait of the uncontrolled and closed-loop motion under $0.3 \mathrm{~g}$ record of the El-Centro earthquake. Also, the spectrum of the first, fourth, sixth, eight and top floor modes for the controlled case is depicted in Fig. 6(b). It can be seen that not only a significant reduction in displacement and velocity trajectories are observed with the proposed controller, but also the system resonant frequencies are shifted further due to the proposed FS2SMC to avoid building collapse from frequency resonance.

Feasibility of the proposed controller is verified via the time responses of the controlled current signals, which are positive and constrained to $2 \mathrm{~A}$, of the MRDs attached to the first and top floors, as plotted in Fig. 7(a). Under the frequency shaped robust control strategy, not only seismic vibrations can be effectively suppressed but also the structural control responses involved can be kept at a low-energy level. Indeed, the conjugate force-displacement trajectory of the MRD under the scaled-down seismic disturbance is depicted in Fig. 7(b), in which $x_{d 1}=x_{1}$ and $f_{\mathrm{MR}(1)}=\Omega^{-1} u_{1}$. The associated kinetic, damping, strain and input energy processes are analyzed in the next section. 


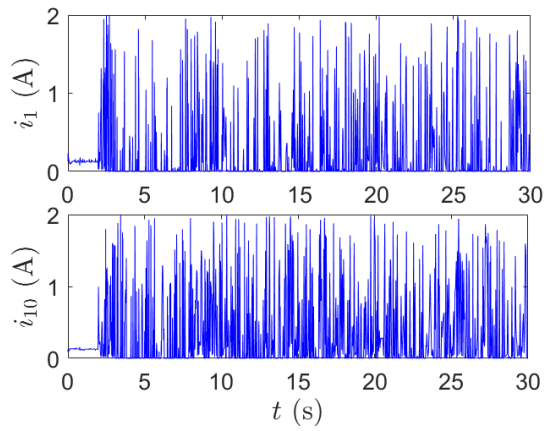

(a)

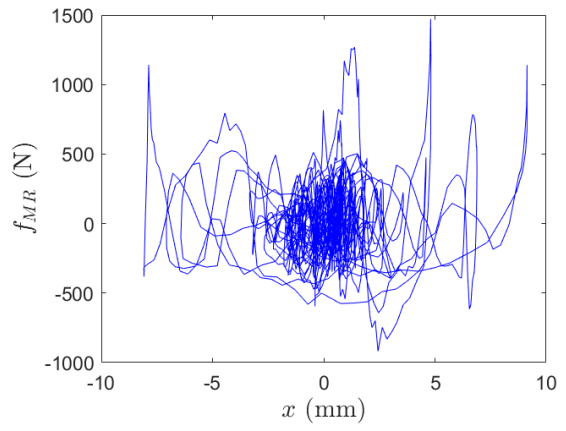

(b)

Figure 7: MRD force-displacement trajectory and applied current.

\section{Discussion}

For analysis of the energy flow in the smart structure, we present in Figs. 8 and 9 the relative kinetic energy (KE), damping energy (DE), strain energy (SE), and input energy (IE) signals with respect to displacement and velocity under $0.3 g$ record of the El-Centro earthquake in both uncontrolled and controlled cases, respectively. Generally, from zero initial conditions $x_{k}\left(t_{0}\right)=\dot{r}_{k}\left(t_{0}\right)=0$, output energy (OE) components, i.e., $E_{k}, E_{\zeta}, E_{s}$, and $E_{i}$ begin to increase from zero when $t \in\left[t_{0} \infty\right)$ under a horizontal ground motion excitation $\ddot{x}_{g}$ starting at $t_{0}$.

In the uncontrolled case, the input energy received from the external disturbance transmitted through the foundation of the smart structural system. The induced energy signal distributes to the kinetic, passive damping, and strain energy of the structure as mechanical OE, i.e. $E_{k}, E_{\zeta}, E_{s}$ and consequently, may exceed a permissible threshold causing structural damage when the magnitude of seismic input is too large. Indeed, due to excessive lateral motion results in structural inability to dissipate the intrinsic $E_{k}, E_{\zeta}, E_{s}$, constituted from the transmitted $E_{i}$ signal, which may eventually lead to serious structural damage.

For the controlled case, the reference is ideally set at $x_{R}=0$ with $L(s)=$ $\frac{1}{s^{2}+1.4142 s+1}, \kappa_{k}=10$, and $\eta_{k}=1.25$. By controlling the capability of absorbing 


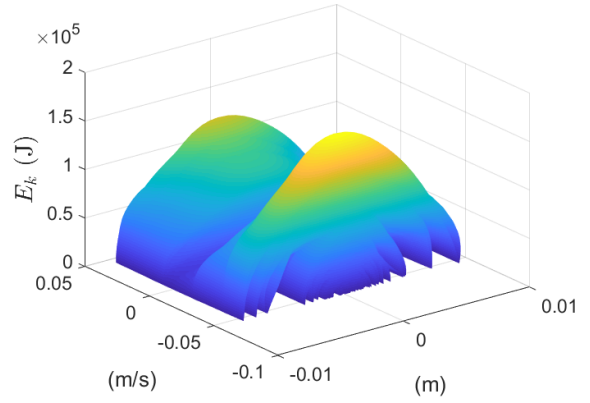

(a) Open-loop $E_{k}$.

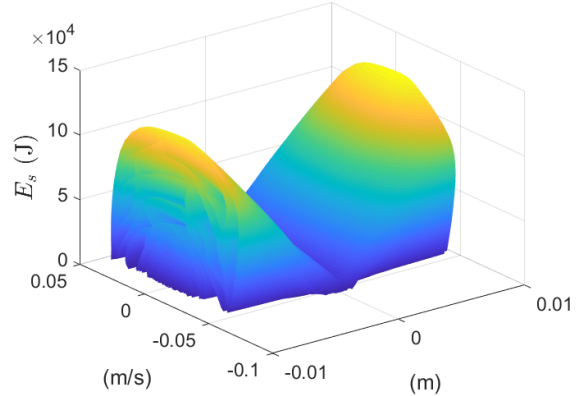

(c) Open-loop $E_{s}$

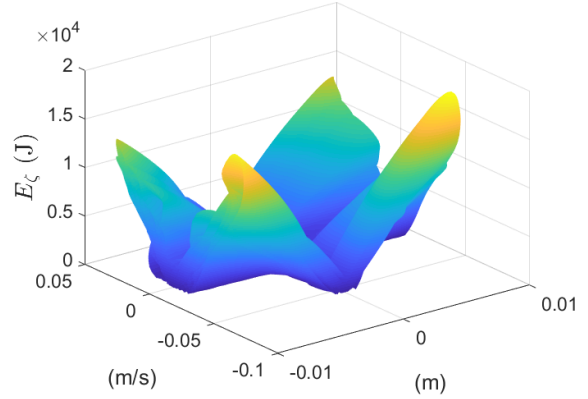

(b) Open-loop $E_{\zeta}$

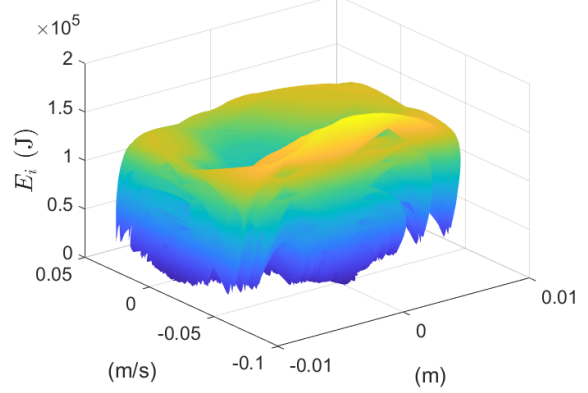

(d) Open-loop $E_{i}$

Figure 8: Uncontrolled relative energy signals under seismic disturbance. 
excitation energy via the use of MR fluid yield stress to ultimately mitigate be seen that for the controlled case, the output energies, namely $E_{k}, E_{\zeta}, E_{s}$ can be substantially reduced more than in the uncontrolled case with MRDs used in the fail-safe passive mode. While both controllers are effective for vibration 


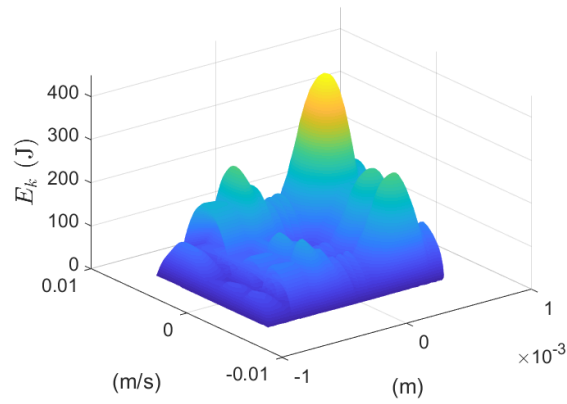

(a) Controlled kinetic energy $E_{k}$.

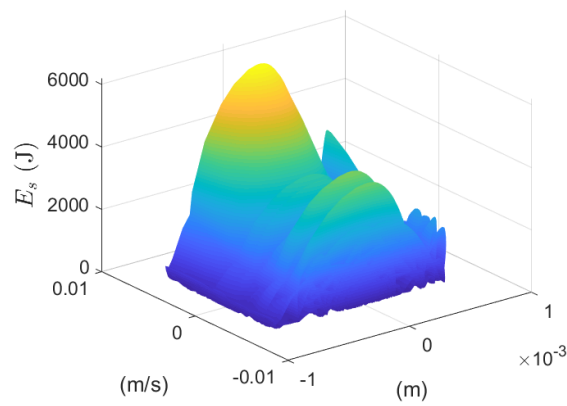

(c) Controlled strain energy $E_{s}$.

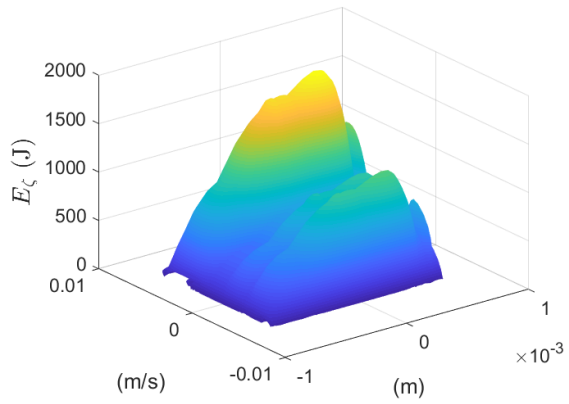

(b) Controlled damping energy $E_{\zeta}$.

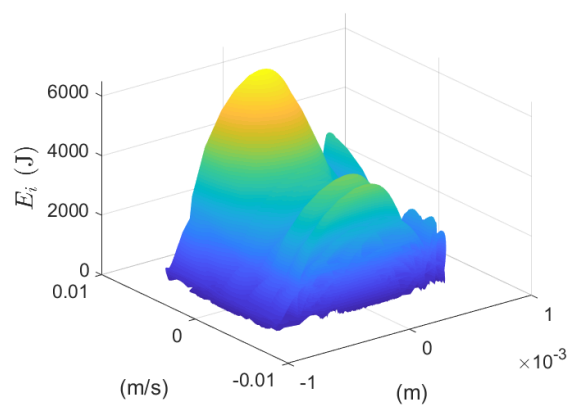

(d) Electrical control energy $E_{c}$.

Figure 9: Controlled relative energy signals under seismic disturbance.

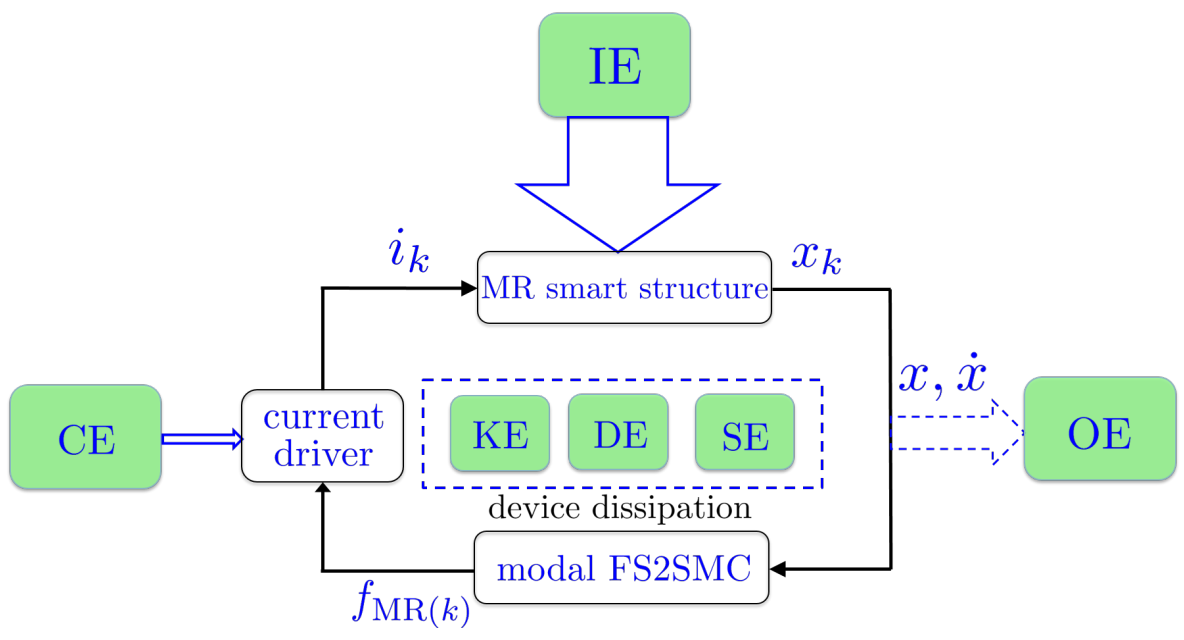

Figure 10: Energy flow in the low-energy smart structure system under external excitation. 
Table 1: Peak relative energy responses $(\mathrm{J})$ under various seismic disturbances.

\begin{tabular}{||c||c|c|c||c|c|c|}
\hline \multicolumn{1}{|c||}{} & \multicolumn{3}{c||}{ El-Centro } & \multicolumn{3}{c|}{ Hachinohe } \\
\cline { 2 - 7 } & UC & LC & FS2SMC & UC & LC & FS2SMC \\
\hline \hline \multirow{2}{*}{$E_{k}$} & $161.9 \times 10^{3}$ & 534.144 & 406.81 & $53.48 \times 10^{3}$ & 137.89 & 106.9 \\
\hline$E_{s}$ & $126.1 \times 10^{3}$ & 8580.7 & 6042.7 & $56.01 \times 10^{3}$ & 2779.7 & 1985.5 \\
\hline$E_{\zeta}$ & $17.1 \times 10^{3}$ & 1865.8 & 1863.9 & 6628.9 & 534.89 & 509.42 \\
\hline$E_{c}$ & - & 8815.1 & 6296.5 & - & 3065.3 & 2114.1 \\
\hline \hline \multicolumn{1}{|c||}{} & \multicolumn{3}{c|}{ Northridge } & \multicolumn{3}{c|}{ Kobe } \\
\cline { 2 - 8 } & UC & LC & FS2SMC & UC & LC & FS2SMC \\
\hline \hline$E_{k}$ & $380.3 \times 10^{3}$ & 2893.8 & 2261.2 & $926.97 \times 10^{3}$ & 1695.4 & 1280.2 \\
\hline$E_{s}$ & $293.8 \times 10^{3}$ & 34143.9 & 29691 & $924.15 \times 10^{3}$ & 40834.9 & 34089 \\
\hline$E_{\zeta}$ & $38.06 \times 10^{3}$ & 11528 & 9606.4 & $112.11 \times 10^{3}$ & 9211.3 & 7676 \\
\hline$E_{c}$ & - & 39074.3 & 31011 & - & 45789 & 35232 \\
\hline
\end{tabular}

300

suppression, the FS2SMC is more energy-efficient than the LC since it requires a smaller amount of control energy $E_{c}$ but results in more absorption of input energy with less stiffness, damping and strain energies at the output. Indeed, owing to the incorporation of the frequency-depending relationships of forcedisplacement and force-velocity of the smart devices into the system model and control design, and hence, the ability to effectively shape the frequency responses of the overall smart structure, the proposed controller can adjust the embodied energy to alter its spectrum in a desired bandwidth, roll off from the resonance region to limit the peak value of the mechanical and transmitted input energy terms, resulting in a low-energy structure while avoiding natural modes of the integrated structural system in dealing with any external loading source.

\section{Conclusion}

We have presented a frequency domain-based method for modeling and control of low-energy structures embedded with smart devices to mitigate the struc- 
tural vibrations and dissipate the energy induced under external excitations. The controlled smart devices, here magnetorheological fluid dampers, are represented by describing functions of amplitude, frequency and control signal. The overall frequency response of the structure is obtained via a modal transformation. A frequency-shaped second-order sliding mode control is then proposed to achieve the control objective of maintaining structural resilience against any dynamic loading sources at a low control energy level. The control signal is a combination of an equivalent control containing a frequency shaping filter, and a robust control to drive the system dynamics to the desired mechanical modes shifted away from the resonance region. Experimental characterization of a laboratory MRD as well as aseismic building structure simulation have been 325 conducted. The structural responses of a 10-floor building subject to benchmark earthquakes and comparison results on kinetic, damping, strain and input energies have indicated effectiveness and feasibility of the proposed method.

\section{Appendix}

Energy dissipated per cyclic oscillation:

Given periodic displacement $x=E \sin (\omega t)$ and velocity $\dot{x}=E \omega \cos (\omega t)$, the energy dissipated by a MRD in one vibration cycle can be determined by the area enclosed within as hysteresis loop, as shown for example in Fig. 2(a):

$$
\begin{aligned}
E_{\mathrm{MR}} & =\oint f_{\mathrm{MR}} d x=\int_{0}^{\frac{2 \pi}{\omega}} f_{\mathrm{MR}} \dot{x} d t=\int_{0}^{\frac{2 \pi}{\omega}} c_{\mathrm{MR}} \dot{x}^{2} d t, \\
& =\frac{c_{\mathrm{MR}}(E \omega)^{2}}{2} \int_{0}^{\frac{2 \pi}{\omega}}(1-\sin (2 \omega t)) d t=2 \pi^{2} f c_{\mathrm{MR}} E^{2},
\end{aligned}
$$
its conjugate variable, the displacement, lying on an ellipse $\left(\frac{f_{\mathrm{MR}}}{c_{\mathrm{MR}} \omega}\right)^{2}+\left(\frac{x}{E}\right)^{2}=1$ depicted in Fig. 11 .

The loss coefficient or damping ratio $\zeta_{\mathrm{MR}}$ of the MRD can be defined as the ratio of damping energy loss per radian divided by the strain energy, i.e., $335 \zeta_{\mathrm{MR}}=\frac{2 \pi^{2} f c_{\mathrm{MR}} E^{2}}{2 \pi\left(k_{\mathrm{MR}} E^{2}\right)}$ where the effective stiffness is $k_{\mathrm{MR}}=\frac{f_{\mathrm{MR}}^{+}-f_{\mathrm{MR}}^{-}}{E^{+}-E^{-}}$. Thus, $c_{\mathrm{MR}}=$ 


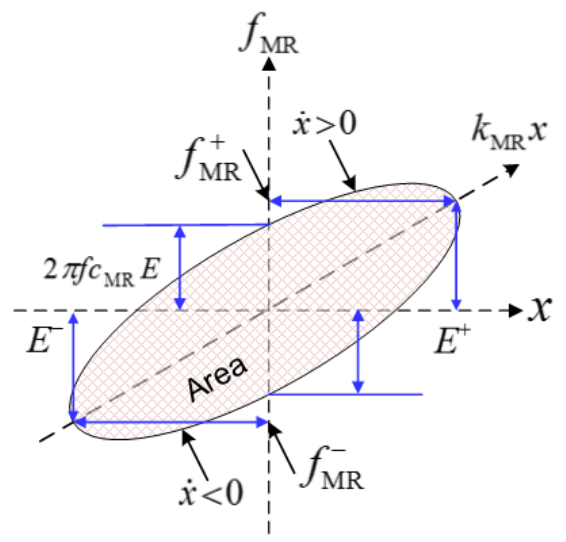

Figure 11: Graphical representation of dissipated-energy per cycle at resonance.

$\frac{k_{\mathrm{MR}} \zeta_{\mathrm{MR}}}{\pi f}$. Using the concept of equivalent viscous damping and substituting $c_{\mathrm{MR}}$ into 23 gives $E_{\mathrm{MR}}=2 \pi k_{\mathrm{MR}} \zeta_{\mathrm{MR}} E^{2}$.

\section{Acknowledgement}

This work is supported by a Distinguished Visiting Scholar scheme at Uni340 versity of Technology Sydney.

\section{References}

[1] I. Sartori and A. Hestnes, "Energy use in the life cycle of conventional and low-energy buildings: a review article" Energy and Buildings, vol. 39, no. 3, pp. 249-257, 2007, doi: 10.1016/j.enbuild.2006.07.001.

${ }_{345}$ [2] M. Liu and B. Mi, "Life cycle cost analysis of energy-efficient buildings subjected to earthquakes," Energy and Buildings, vol. 154, pp. 581-589, 2017, doi: 10.1016/j.enbuild.2017.08.056.

[3] A. Belleri and A. Marini, "Does seismic risk affect the environmental impact of existing buildings?" Energy and Buildings, vol. 110, pp. 149-158, 2016, doi: 10.1016/j.enbuild.2015.10.048. 
[4] Z. Nadoushani and A. Akbarnezhad, "Effects of structural system on the life cycle carbon footprint of buildings," Energy and Buildings, vol. 102, pp. 337-346, 2015, doi: 10.1016/j.enbuild.2015.05.044.

[5] A. Akbarnezhad and J. Xiao, "Estimation and minimization of embodied carbon of buildings: a review," Buildings, vol. 7, no. 1, 2017, doi: 10.3390/buildings7010005.

[6] T. Alqado, G. Nikolakopoulos and J. Jonasson, "Comfort level identification for irregular multi-storey building," Automation in Construction, vol. 50, pp. 40-49, 2015, doi: 10.1016/j.autcon.2014.10.009.

[7] A. K. Chopra and F. McKenna, "Modeling viscous damping in nonlinear response history analysis of buildings for earthquake excitation," Earthquake Engineering and Structural Dynamics, vol. 45, pp. 193-211, 2016, doi: 10.1002/eqe.2622.

[8] F. Ikhouane, V. Manosa, and J. Rodellar, "Adaptive control of a hysteretic structural system," Automatica, vol. 41, pp. 225-231, 2005, doi: 10.1016/j.automatica.2004.08.018.

[9] M. M. Ali and K. S. Moon, "Structural developments in tall buildings: current trends and future prospects," Architectural Science Review, vol. 50, no. 3, pp. 205-223, 2007, doi:10.3763/asre.2007.5027.

[10] R. Smith, R. Merello, and M. Willford, "Intrinsic and supplementary damping in tall buildings" Proc. of the Institution of Civil Engineers: Structures and Buildings, vol. 163, no. 2, pp. 111-118, 2010, doi: 10.1680/stbu.2010.163.2.111.

[11] Q. P. Ha, M. T. Nguyen, J. Li, and N. M. Kwok, "Smart structures with current-driven MR dampers: modelling and second-order sliding mode control," IEEE/ASME Trans. on Mech., vol. 18, no. 6, pp. 1702-1711, 2013, doi: 10.1109/TMECH.2013.2280282. 
[12] Q. P. Ha, N. M. Kwok, M. T. Nguyen, J. Li, and B. Samali, "Mitigation of seismic responses of building structures using MR dampers with lyapunovbased control," Structural Control and Health Monitoring, vol. 15, no. 6, pp. 604-621, 2008, doi: 10.1002/stc.218.

[13] Q. P. Ha, S. Royel, J. Li, and Y. Li, "Hysteresis modeling of smart structure MR devices using describing functions," IEEE/ASME Trans. on Mechatronics, vol. 21, no. 1, pp. 44-50, 2016, doi: 10.1109/TMECH.2015.2506539.

[14] C. Uang and V. Bertero, "Evaluation of seismic energy in structures," Earthquake Engineering and Structural Dynamics, vol. 19, pp. 77-90, 1990, doi: $10.1002 /$ eqe.4290190108.

[15] J. E. Luco, "A note on classical damping matrices," Earthquake Engineering and Structural Dynamics, vol. 37, pp. 615-626, 2008, doi: 10.1002/eqe.776.

390 [16] H. Li, and J. Wang, "Experimental investigation of the seismic control of a nonlinear soil-structure system using MR dampers," Smart Materials and Structures, 20(8):085026, 2011, doi:10.1088/0964-1726/20/8/085026.

[17] F. Weber, H. Distl, and M. Motavalli, "Cycle energy control of magnetorheological dampers on cables," Smart Materials and Structures, vol. 18, pp. 1123-1149, 2009, doi: 10.1088/0964-1726/18/1/015005.

[18] J. Hogsberg and A. Krenk "Energy dissipation control of magnetorheological damper," Probabilistic Engineering Mechanics, vol. 23, pp.188-197, 2008, doi: 10.1016/j.probengmech.2007.12.007.

[19] F. Gordaninejad and D. Breese, "Heating of magnetorheological fluid dampers," Journal of Intelligent Material Systems and Structures, vol. 10, pp. 634-645, 1999, doi: 10.1106/55D1-XAXP-YFH6-B2FB.

[20] R. Zalewski, J. Nachman, M. Shillor, and J. Bajkowski, "Dynamic model for a magnetorheological damper," Applied Mathematical Modelling, vol. 38, pp. 2366-2376, 2014, doi: 10.1016/j.apm.2013.10.050. 
[21] N. K. Gupta, "Frequency-shaped cost functionals: extension of linearquadratic-Gaussian design methods," Journal of Guidance, Control and Dynamics, vol. 3, no. 6, pp. 529-535, 1980, doi: 10.2514/3.19722.

[22] K. C. Cheok, H. X. Hu, and N. K. Loh, "Discrete-time frequency-shaping parametric LQ control with application to active seat suspension control," IEEE Transactions on Industrial Electronics, vol. 36, no. 3, pp. 383-390, 1989, doi: 10.1109/41.31501.

[23] J. J. E. Slotine and W. Li, Applied Nonlinear Control, Prentice-Hall, Englewood Cliffs, New Jersey, 1991.

[24] H. K. Khalil, Nonlinear Systems, 3rd ed., NJ, USA: Prentice Hall, 2002.

[25] Y. Shtessel, C. Edwards, L. Fridman and A. Levant, Sliding Mode Control and Observation, Birkhauser Boston Inc, 2014.

[26] J. B. Moore and D. L. Minigori, "Robust frequency-shaped LQ control," Automatica, vol. 23, no.5, pp. 641-645, 1987, doi: 10.1016/00051098(87)90060-4.

[27] A. Koshkouei and A. Zinober, "Robust frequency shaping sliding mode control," IEE Proceedings - Control Theory and Applications, vol. 147, no. 3, pp. 312-320, 2000, doi: 10.1049/ip-cta:20000378.

[28] L. Zuo and J. Slotine, "Robust vibration isolation via frequency-shaped sliding control and modal decomposition," Journal of Sound and Vibration, vol. 285, pp. 1123-1149, 2005, doi: 10.1016/j.jsv.2004.09.014.

[29] M. Zheng and M. Tomizuka, "A frequency-shaping methodology for discrete-time sliding mode control," International Journal of Control, 2017, doi: 10.1080/00207179.2017.1406148.

[30] T. Acarman and U. Ozguner, "Frequency shaping compensation for back430 stepping sliding mode control," IFAC Proceedings Volumes, vol. 35, no. 1, pp. 37-42, 2002, doi: 10.3182/20020721-6-ES-1901.01077. 
[31] A. Mehta and B. Bandyopadhay, "Frequency-shaped sliding mode control using output sampled measurements," IEEE Transactions on Industrial Electronics, vol. 56, no. 1, pp. 28-35, 2009, doi: 10.1109/TIE.2008.2006946.

[32] C. Schwartz and R. Gran, "Describing function analysis using MATLAB and Simulink," IEEE Control Systems Magazine, vol. 21, no. 9, pp. 19-26, 2001, doi: 10.1109/37.939940.

[33] G. Bartolini, A. Ferrara, and E. Usai, "Chattering avoidance by secondOrder sliding mode control," IEEE Transactions on Automatic Control, vol. 43, no. 2, pp. 241-246, 1998, doi: 10.1109/9.661074.

[34] A. Levant, "Principles of 2-sliding mode design," Automatica, vol. 43, no.4, pp. 576-586, 2007, doi: 10.1016/j.automatica.2006.10.008.

[35] M. Hadi, and Y. Arfiadi, "Optimum design of absorber for MDOF structures," Journal of Structural Engineering, vol. 124, no. 11, pp. 1272-1280, 1998, doi: 10.1061/(ASCE)0733-9445(1998)124:11(1272). 